\title{
EUV-multilayers on grating-like topographies
}

\author{
A. J. R. van de Boogaard, ${ }^{1,}$ E. Louis, ${ }^{1}$ K. A. Goldberg, ${ }^{2}$ I. Mochi, ${ }^{2}$ and F. Bijkerk ${ }^{1,3}$ \\ 'FOM Institute for Plasma Physics Rijnhuizen, P.O.Box 1207, 3430 BE, Nieuwegein, The Netherlands \\ ${ }^{2}$ Center for X-Ray Optics, Lawrence Berkeley National Laboratory, Berkeley, CA 94720, USA \\ ${ }^{3}$ MESA+ Institute for Nanotechnology, University of Twente, P.O. Box 217 , \\ 7500 AE Enschede, The Netherlands
}

In this study, multilayer morphology near the key anomalies in grating-like structures, namely sharp step-edges and steep walls, are examined. Different deposition schemes are employed. Based on cross section TEM analysis an explanatory model describing the morphology of the successive layers is developed. A further insight into the periodicity and the general performance of the multilayer is obtained by EUV microscopy. The main distortions in multilayer structure and hence EUV performance are found to be restricted to a region within a few hundred nanometers from the anomalies, which is very small compared to the proposed grating period $(50-100 \mu \mathrm{m})$. These multilayer coated blazed gratings can thus be considered a viable option for spectral purity enhancement of EUV light sources.

Keywords: Mo/Si Multilayers, Blazed Grating, Spectral Purity, EUV Source, Transmission Electron Microscopy, EUV microscopy

\section{INTRODUCTION}

For the spectral separation of EUV and parasitic IR light, both critically present in the overall spectrum of $\mathrm{CO} 2$ laser driven Laser Produced Plasma sources, special multilayer-coated reflection gratings show great potential. Based on rigorous calculations is has been demonstrated that full spectral separation can be obtained from $\mathrm{Mo} / \mathrm{Si}$ multilayers deposited on a blazed grating, provided the grating topography can be replicated by the multilayer stack.' Unlike other approaches based onto antireflective layers, ${ }^{2}$ transmitive filtering, ${ }^{3}$ or grid-type filters ${ }^{4}$ our grating based method is not coming at the expense of EUV throughput.

The use of the blazed grating acting as an EUV multilayer substrate, introduces tight margins on the quality of the general grating design and on the roughness of the facets in particular. State-of-the-art blazed gratings designed for IR-diffraction with soft $x$-ray specifications on roughness (rms roughness values around $I \mathrm{~nm}$ ), are commercially available but too expensive to serve as test system for proof-ofprinciple studies. For this reason, the basic phenomena of multilayer deposition on grating-like topographies are studied using a model system, which mimics the key features of a blazed grating.

\section{EXPERIMENTS}

The blazed grating surface has a saw-tooth profile in one dimension of the surface plane ( $\mathrm{x}$ direction, fig. 1), which is defined by the period $\lambda$, the blaze-angle $\theta$, and the apex-angle $\phi$. For the proposed grating period $\lambda=50-100 \mathrm{~nm}$ and $\theta \approx 10^{\circ}$ and $\phi \approx 90^{\circ}$, the individual grating facets are considered as infinitely wide and high compared to the typically $350 \mathrm{~nm}$ thick Mo/Si multilayer structure. We have used cleaved superpolished c-Si (100) wafers (fig. lb) as a model system of an individual grating facet (fig. l.a). The cleaving process produces reproducible, sharp and well defined

"Corresponding author: a.j.r.vandenboograarda a rijnhuizen.nl 
wafer edges. Secondly. we used a strip of mica foil, which crystallinity results in a stepped-terrace grating-like topography at the edge (fig.1.c). The terraces are tens of $\mu \mathrm{m}$ wide and approximately one $\mu \mathrm{m}$ high. On both samples Mo/Si multilayer systems, optimized for near-normal EUV reflectance, were deposited to study the multilayer morphology near the sharp step-edges and vertical walls.
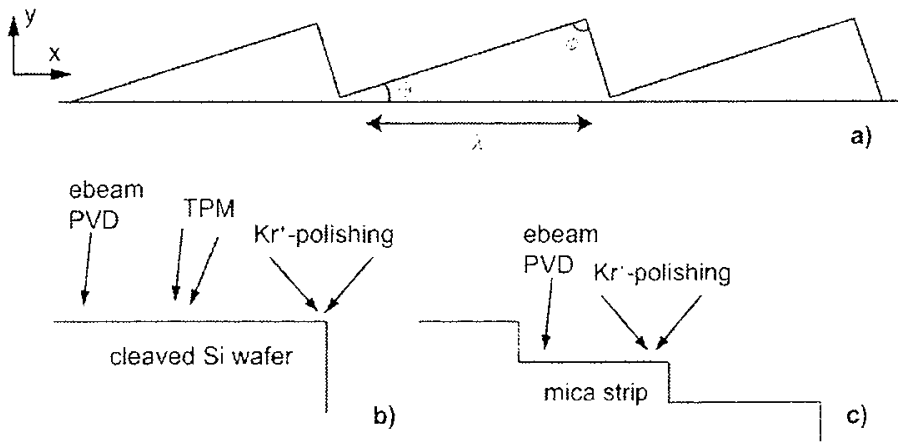

Fig. 1) Schematic diagram of a) blazed grating b) cleaved Si wafer and deposition geometry c) mica strip and deposition geometry

The samples were mounted on a rotating substrate holder in the FOM coating facility. ${ }^{5}$ Both ebeam physical vapour deposition (PVD) as a thermalized particle magnetron deposition ${ }^{6}$ (TPM) were employed in individual experiments for the deposition the multilayers on the cleaved wafers. For the mica sample, e-beam PVD was performed only. At both the e-beam PVD and TPM deposition experiments, a low energy $\mathrm{Kr}^{+}$-ion polishing treatment was applied for surface smoothing after grow'th of each Si layer The Kaufmann ion-source was mounted under an angle of approximately $45^{\circ}$ with respect to the axis of substrate rotation.

\section{RESULTS}

\section{Cross-section Transmission Electron Microscopy}

To study the morphology evolution in the sequentially deposited Mo/Si layers. cross-section transmission electron microscopy (cs-TEM) was used (Fig.2.a-c). On the cleaved wafer samples (Fig.2.a and b). it is clearly visible that the Mo/Si layered structure is well defined and semi-parallel, up to the sample-edge. The multilayer structure is extending the step-edge by approximately $50 \mathrm{~nm}$; the overhang is larger than expected based on deposition geometry and might be due to lateral re-

distributing of deposited lay'er material during ion-polishing. Furthermore, a profound rounding of the sharp wafer edge by the overhanging successive layers is observed, increasing with the layer number. The slope of the cleaved wafer and $\mathrm{Mo}$ /Si multilayer structure are acting as a substrate for the overhanging multilayer material, resulting in a more disordered morphology which is associated with the locally very small grazing angle of incidence of the deposition plume. Here the lateral thickness is estimated on approximately $100-150 \mathrm{~nm}$

On the mica sample, the multilayer characteristics at a terrace near the step-edge, are similar as for the cleaved Si wafers. Near the steep ridge and convex corner with an underlying terrace (Fig.2.c). a sharp transition between disordered multilayer morphology and well defined. semi-parallel layers is observed, without the strong rounding of the edge as observed at the step-edges. This transition occurs within $50 \mathrm{~nm}$ from the convex corner. 


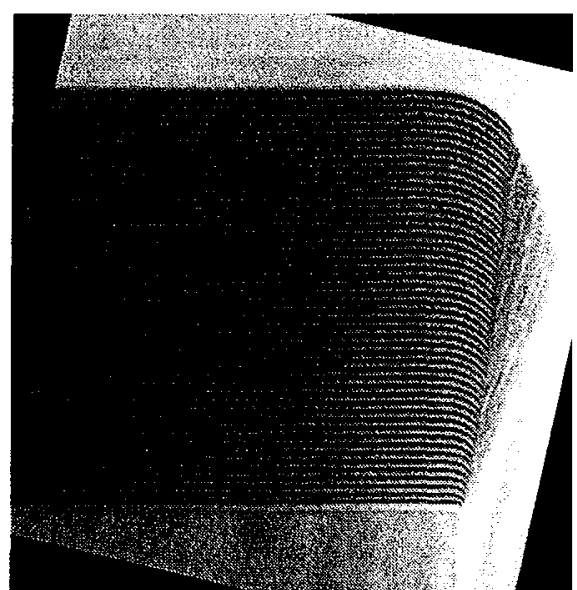

a)

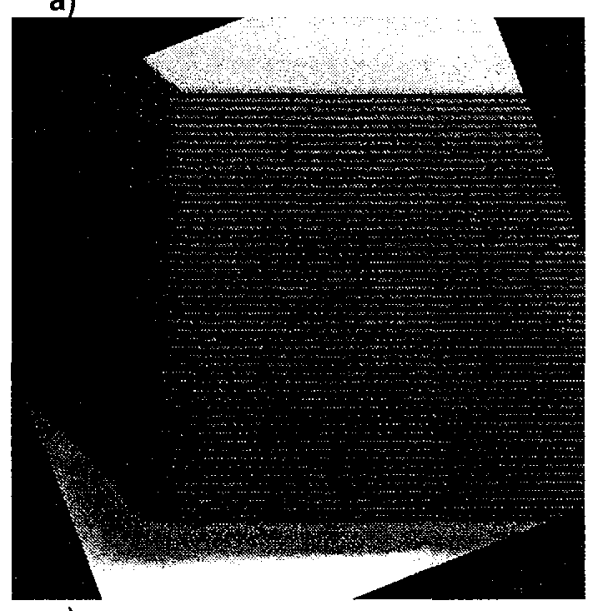

c)

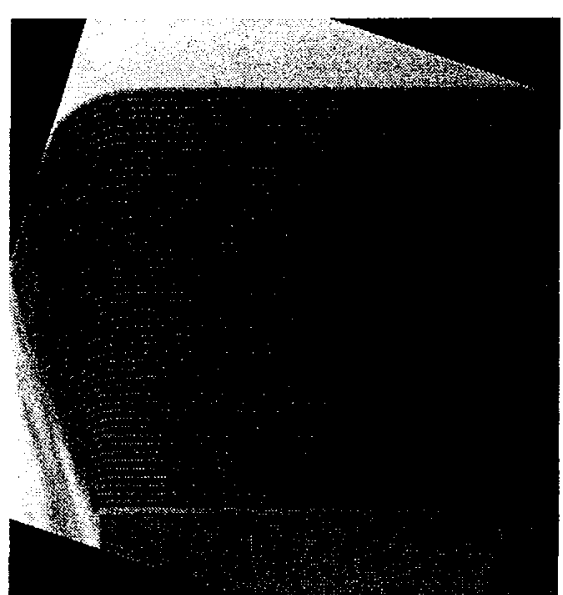

b)

Fig. 2) cs-TEM images of $50 x 7 \mathrm{~nm}$.Mo:Si multilayer structures, black frames are trivial a) e-beam PVD on cleaved wafer b) TPM deposition on cleaved wafer $c$ ) e-beam PVD on mica. comex corner.

It is found that the step-edge and the underlying convex corner enclose the region showing the main deviations from the semi-parallel layered structure. To obtain a further insight into the details of the multilayer structure in the semi-parallel regime, a simplified continuum-model of layer profile $(h)$ evolution (1) can be applied since large surface slopes are absent;

$$
\begin{aligned}
& \frac{\partial}{\partial t}|h(q, t)|^{2}=-b(q) \cdot|h(q, t)|^{2}, \\
& b(q)=\sum_{1} v, q^{\prime},
\end{aligned}
$$

with $t$ the thickness, $q$ the spatial frequency, and $v$, a proportionality coefficient. The relevant layer distributing kinetics (e.g. during ion-polishing) are linear in reciprocal space. A comparison of the calculated and measured (cs-TEM) average d-spacing is shown in (Fig.3), and hints for dominant smoothing kinetics $\propto q$ with proportionality constants for the e-beam PVD and TPM deposition of $v=$ 0.1 and $v=0.6$ respectively. The difference may be related to the deposition conditions. For the e-beam PVD $\left(E_{k i n}=0.1 \mathrm{eV}\right.$ ) layers, the smoothing is induced by the ion-polishing treatment. Sputtering of the target material as employed during TPM deposition, causes a more energetic deposition $\left(E_{\mathrm{km}} \approx 3 \mathrm{eV}\right.$ ) and, most likely, an additional ion-fluence at the samples during deposition, giving rise to enhanced smoothing kinetics. The offset in d-spacing for the multilayer structure near the convex corner is 
considered to be due to partial shadowing from the ion beam, and might be prevented by adjusting deposition geometry. A more advanced model, also taking the non-linear kinetics into account, is currently developed and results will be published elsewhere.

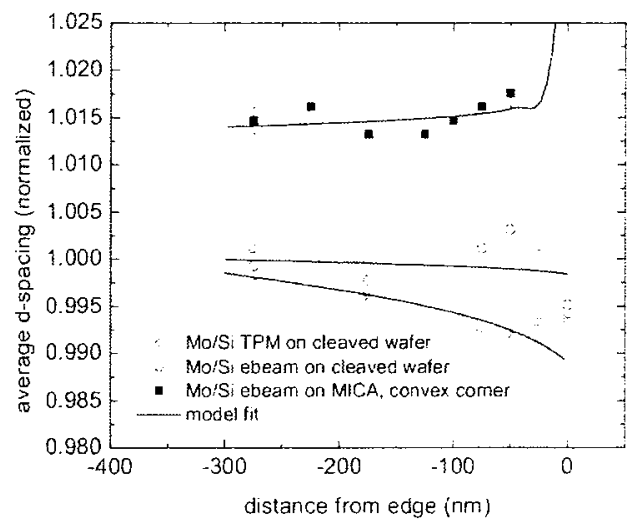

Fig. 3) Average d-spacing near step-edge and convex corner. CS TEM data and calculations are shown.

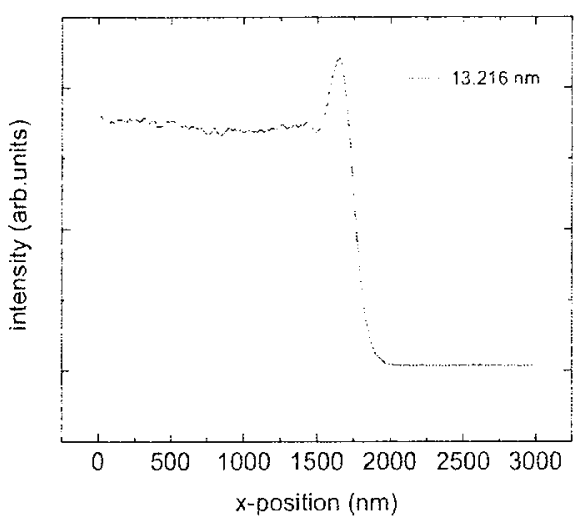

Fig. 4) Reflected intensity profile of e-beam PVD Mo'Si multilayer near step-edge.

Actinic Inspection Tool

Additional to cS-TEM, the multilayer structure near the step-edges at the cleaved wafers was studied by EUV microscopy measurements, using the actinic inspection tool (AIT) facility at the Centre for X-ray Optics, Lawrence Berkeley National Laboratory. The wavelength-dependence in the focal length of the imaging zoneplate was exploited to adjust the AIT focus relative to the sample under study. ${ }^{7}$ The intensity of the thus obtained in-focus images is proportional to the local EL' $V$ reflectance with a lateral resolution of approximately $100 \mathrm{~nm}$, but the AIT is not calibrated to give an absolute value of the measured reflectance. Intensity profiles were taken perpendicular to the cleaving edge: an example is shown in (fig.4.). It is found that the multilayer reflectance is stationary over a lateral distance of $1500 \mathrm{~nm}$. From this point the signal increases with $30 \%$ over $200 \mathrm{~nm}$ and drops off to noise level over the next $200 \mathrm{~nm}$. The stationary reflectance values give a robust indication of an unmodified multilayer structure. No definite edge position of the substrate under the multilayer can be derived from the data but the stationary reflectance approaches the edge up to a distance of $400 \mathrm{~nm}$ at maximum, taking the zero intensity as outermost possible position of the edge. The subsequent increase of reflectance is associated with a decreasing d-spacing in the semi-parallel multilayer regime, as in agreement with the TEM observations.

\section{CONCLUSIONS}

Mo/Si multilayer structures deposited onto substrates with a sharp cut-off, have been demonstrated to maintain their layered properties up to the step edge. This result enables the fabrication of multilayer coated IR grating geometries, which allow the full spatial separation of undesirable IR light components in EUV beams, without any EUV losses.

In our experiments, multilayers deposited by e-beam PVD and TPM show a qualitatively similar behavior. On substrates with a terrace-like structure, the e-beam PVD multilayers show a transition from the disordered morphology on the steep ridge to the semi-parallel regime on underlying terrace within $50 \mathrm{~nm}$, without the profound rounding of the (convex) edge as observed at the step-edge. At the semi-parallel regime, the $d$-spacing evolution for both substrate topographies can be described by a linear model of surface height-profile evolution as a function of layer thickness. Best substrate 
replication is obtained by e-beam PVD. First EUV microscopy data is in agreement with the cs-TEM observations, estimating the edge effect in the semi-parallel multilayer regime to be restricted to 200 $\mathrm{nm}$ and the total edge-effect to be restricted to $400 \mathrm{~nm}$. Relative to a grating period of $50-100 \mu \mathrm{m}$, this is considered to be well within budget for allowing the application as EUV lossless, wavelength selective filtering elements.

\section{ACKNOWLEDGEMENTS}

We acknowledge financial support from SenterNovem through the EXEPT and EAGLE programmes co-ordinated by ASML and the Foundation for Fundamental Research on Matter (Stichting voor Fundamenteel Onderzoek der Materie, FOM) and Carl Zeiss SMT AG through the Industrial PArtnership Programme XMO.

Furthermore the authors wish to thank E.G. Keim for the TEM analysis, and B. La Fontaine for making experimental time available on the AIT.

Supported by the U.S. Department of Energy under Contract No. DE-AC02-05CH11231.

$'$ A. J. R. van den Boogaard, E. Louis, F. A. van Goor, and F. Bijkerk, Proc. SPIE 7271, 72713B (2009).

${ }^{2}$ W. A. Soer, P. Gawlitza, M. M. J. W. van Herpen, M. J. J. Jak, S. Braun, P. Muys, and V. Y. Banine, Opt. Lett, 34, 3680 (2009).

${ }^{3}$ M. S. Bibishkin, N. I. Chkhalo, S. A. Gusev, E. B.Kluenkov, A. Y. Lopatin, V. I. Luchin, A. E. Pestov, N.

N. Salashchenko, L. A. Shmaenok, N. N. Tsybin, and S. Y. Zuev, Proc. SPIE 7025, 702502 (2008).

${ }^{4}$ W. A. Soer, M. J. J. Jak, A. M. Yakunin, M. M. J. W. Herpen, and V. Y. Banine, Proc. SPIE 7271, $72712 Y$ (2009).

${ }^{5}$ E. Louis, F. Bijkerk, L. Shmaenok, H. -J. Voorma, M. J. van der Wiel, R. Schlatmann, J. Verhoeven, E. W. J. M. van der Drift, J. Romijn, B. A. C. Rousseeuw, F. Voss, R. Dresor, and B. Nikolaus, Microelectron. Eng. 21, 67 (1993).

${ }^{6}$ A.E. Yakshin, R.W.E. van de Kruijs. I. Nedelcu, E. Zoethout, E. Louis, F. Bijkerk, H. Enkisch and S. Müllender, Proc. SPIE 6517,65/701 (200?).

${ }^{7}$ K. A. Goldberg, I. Mochi, S. Huh, Proc. SPIE 7271, 72713N (2009). 